\title{
Syntheses of fluorooxindole and 2-fluoro-2-arylacetic acid derivatives from diethyl 2-fluoromalonate ester
}

\author{
Antal Harsanyi ${ }^{1}$, Graham Sandford ${ }^{* 1}$, Dmitri S. Yufit ${ }^{2}$ \\ and Judith A.K. Howard ${ }^{2}$
}

\author{
Full Research Paper \\ Address: \\ ${ }^{1}$ Department of Chemistry, Durham University, South Road, Durham, \\ DH1 3LE, UK and ${ }^{2}$ Chemical Crystallography, Department of \\ Chemistry, Durham University, South Road, Durham, DH1 3LE, UK \\ Email: \\ Graham Sandford* - graham.sandford@durham.ac.uk \\ * Corresponding author \\ Keywords: \\ fluorinated building blocks; fluoroarylacetic acid; fluoromalonate; \\ fluorooxindole; organo-fluorine; selective fluorination
}

Open Access

Beilstein J. Org. Chem. 2014, 10, 1213-1219.

doi:10.3762/bjoc.10.119

Received: 10 January 2014

Accepted: 16 May 2014

Published: 22 May 2014

This article is part of the Thematic Series "Organo-fluorine chemistry III".

Guest Editor: D. O'Hagan

() 2014 Harsanyi et al; licensee Beilstein-Institut. License and terms: see end of document.

\begin{abstract}
Diethyl 2-fluoromalonate ester is utilised as a building block for the synthesis of 2-fluoro-2-arylacetic acid and fluorooxindole derivatives by a strategy involving nucleophilic aromatic substitution reactions with ortho-fluoronitrobenzene substrates followed by decarboxylation, esterification and reductive cyclisation processes.
\end{abstract}

\section{Introduction}

Since 1954, when Fried and Sabo observed that the incorporation of a fluorine atom into a corticosteroid derivative led to valuable enhanced biological activity [1], a growing number of commercially significant life science products, which owe their activity to the presence of fluorine atoms within their structures, have developed. Fluorine incorporation can lead, for example, to enhanced bioavailability, metabolic stability and lipophilicity of the organic system and these properties are exploited in a number of commercially valuable drugs including Ciprofloxacin, Lipitor and Voriconazole [2-6].

Given the very small number of fluorinated systems available from nature [7-9], in essence all organic molecules bearing carbon-fluorine bonds are 'man-made'. Syntheses rely either on the construction of carbon-fluorine bonds using a fluorinating agent ('late-stage' fluorination) or the application of polyfunctional fluorine-containing small molecule building blocks ('early stage' fluorination) which may be employed in further transformations involving all the reactions and techniques available to synthetic organic chemists [10-13]. Of course, the success of an 'early stage' fluorination approach depends on the availability of a range of appropriately functionalised, fluorinated building blocks and the establishment of corresponding reactivity profiles [14]. However, it does not necessarily follow that reactions for which regio- and stereoselectivity profiles are well established for hydrocarbon systems will be similar to those for corresponding selectively fluorinated systems and, indeed, this is often not the case [15]. 
The use of 1,3-diketone, 1,3-ketoester and 1,3-diester derivatives in retrosynthetic planning is widespread in general organic chemistry and numerous terpenes, heterocycles and steroids originate from such simple yet synthetically versatile substrates [16-19]. In contrast, despite the availability of synthetic procedures for the preparation of various 2-fluoro-1,3-dicarbonyl systems [20-27], there is, surprisingly, only a relatively limited number of publications that report the use of such potentially useful fluorinated building blocks for the synthesis of more structurally sophisticated selectively fluorinated systems. For example, 2-fluoromalonate esters have been used for the preparation of various $\alpha$-fluorocarboxylic acids [28-32], heterocycles, such as fluoropyrimidine [33] and quinolone [34] derivatives, alkylated [35] and Michael addition [36-40] products, providing an indication of the potential uses and opportunities available for the synthesis of fluoro-organic products from fluoromalonate precursors.

As part of a wider research programme aimed at developing routes for the synthesis of selectively fluorinated molecules using elemental fluorine for the key construction of the carbon-fluorine bond by complementary direct selective direct fluorination [41-44], continuous flow [45-49] and building block [50] strategies, in this paper, we describe nucleophilic aromatic substitution reactions of carbanions derived from diethyl 2-fluoromalonate ester as the first stage in the synthesis of fluoroacetic acid and fluoroxindole systems. While related palladium catalysed coupling processes between aryl bromides and diethyl 2-fluoromalonate have been described [51], reactions involving nucleophilic aromatic substitution between fluoromalonate systems [52] and appropriate aryl substrates have not been reported previously. Recently, various routes to fluorooxindoles have been discussed involving enantioselective fluorination of appropriate oxindole substrates by electrophilic fluorinating agents [53-62] or DAST [63] providing an indication of the importance of fluorooxindoles for medicinal chemistry applications.

\section{Results and Discussion}

Reactions of carbanions generated by the addition of sodium hydride to a solution of diethyl 2-fluoromalonate (1) in DMF with ortho-fluoronitrobenzene (2a) led to the efficient displacement of fluorine by a nucleophilic aromatic substitution process to provide diester 3 in good yield (Scheme 1). Displacement of fluorine from ortho-fluoronitrobenzene was quantitative as measured by ${ }^{19} \mathrm{~F}$ NMR spectroscopy of the crude reaction mixture and the structure of isolated diester $\mathbf{3}$ was confirmed by X-ray crystallography (Figure 1).

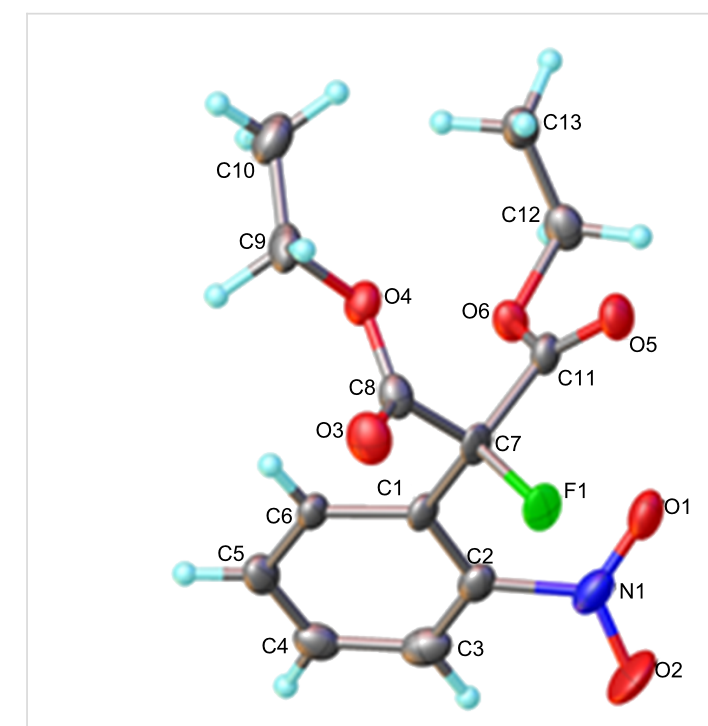

Figure 1: Molecular structure of 3

In initial experiments, decarboxylation of $\mathbf{3}$ by reaction with potassium hydroxide gave good yields of the corresponding 2-fluoro-2-arylacetic acid 4a. However, in subsequent experiments, we found that further purification of the diester $\mathbf{3}$ after the initial $S_{N} A r$ step was not necessary and decarboxylation of crude diester 3 gave 4a very efficiently. Consequently, in all analogous experiments (Table 1), crude product diesters of type 3 were isolated and used without further purification, allowing the ready synthesis of a range of arylfluoroacetic acid derivatives 4a-f (Table 1). Structures 4a-f were confirmed by NMR techniques and, in particular, a doublet located at $-190 \mathrm{ppm}$ $\left({ }^{2} J_{\mathrm{HF}}=50 \mathrm{~Hz}\right)$ in the ${ }^{19} \mathrm{~F}$ NMR spectra assigned to the $\mathrm{CFH}$ resonances and the corresponding doublets observed at $\sim 6 \mathrm{ppm}$ in the ${ }^{1} \mathrm{H}$ NMR spectra, are diagnostic for the structures proposed.

A nitro group ortho to a fluorine atom on the aryl ring is necessary under the present conditions to achieve full conversion of<smiles>CCOC(=O)C(F)C(=O)OCC</smiles><smiles>O=[N+]([O-])c1ccccc1F</smiles>

$2 a$

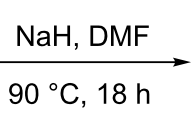<smiles>CCOC(=O)C(F)(C(=O)OCC)c1ccccc1[N+](=O)[O-]</smiles>

$3,71 \%$ 
Table 1: $S_{N} A r$ reactions using fluoromalonate derivatives.<smiles>CCOC(=O)C(F)C(=O)OCC</smiles>

1<smiles>[X]c1cccc(F)c1[N+](=O)[O-]</smiles>

2a-f
1) $\mathrm{NaH}, \mathrm{DMF}$

2) $\mathrm{KOH}, \mathrm{MeOH}$

4a-f

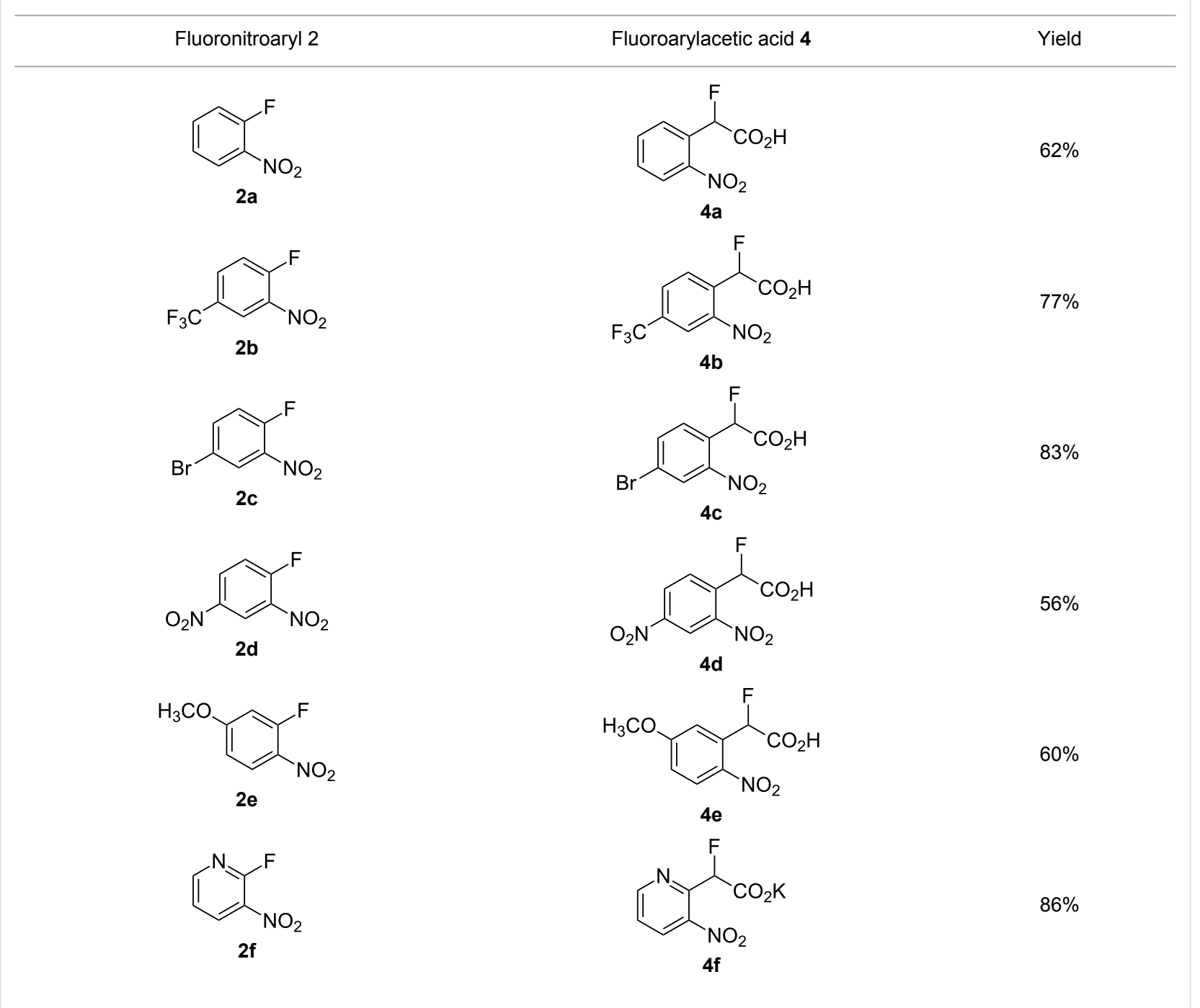

the starting fluoroarene. In related experiments, we found that a para-trifluoromethyl group is not sufficiently activating for reaction to occur whilst para-fluoronitrobenzene gave a complex mixture of unidentified products, most probably derived from competing benzyne formation.

This efficient methodology complements reported processes for the synthesis of various biologically active 2-fluoro-2-phenylacetic acids [64] which may be prepared using electrophilic fluorination of enolate esters [64-66], deoxofluorination [67-69] nucleophilic [70] and electrochemical fluorination [71,72] strategies.
Attempts to prepare 2-fluoro-2-(2,4-dinitrophenyl)acetic acid by an analogous process led to the isolation of a benzyl fluoride derivative 5, after evaporation of toluene and purification by column chromatography in $61 \%$ yield. The two consecutive decarboxylation reactions reflect the greater stability of the benzylic carbanion formed on loss of carbon dioxide from this system (Scheme 2).

With the series of 2-fluorophenylacetic acids in hand, we attempted the reduction of the nitro group in $\mathbf{4 a}$ using sodium dithionite, adapting reaction conditions similar to those described in the literature for the synthesis of biologically active 
<smiles>O=C(O)C(F)c1ccc([N+](=O)[O-])cc1[N+](=O)[O-]</smiles>

Scheme 2: Synthesis of benzyl fluoride derivative $\mathbf{5}$.

system MaxiPost [63]. However, very low isolated yields of the cyclised product were obtained, presumably because of the high solubility of the amino acid intermediate in the aqueous reaction mixture and the well-established difficulty of direct amide bond formation processes. Consequently, before carrying out the nitro group reduction and amide forming cyclisation reactions, the acids $\mathbf{4 a - e}$ were transformed to the corresponding methyl esters $\mathbf{6 a}-\mathbf{e}$ by stirring a mixture of the acid in hydrochloric acid and methanol (Table 2). The structure of $\mathbf{6 a}$

Table 2: Synthesis of methyl ester derivatives.

$$
\begin{aligned}
& { }_{\mathrm{NO}_{2}}^{\mathrm{CO}_{2} \mathrm{H}} \underset{70{ }^{\circ} \mathrm{C}, 16 \mathrm{~h}}{\mathrm{HCl}, \mathrm{MeOH}} \\
& \text { 4a-e } 6 a-e
\end{aligned}
$$

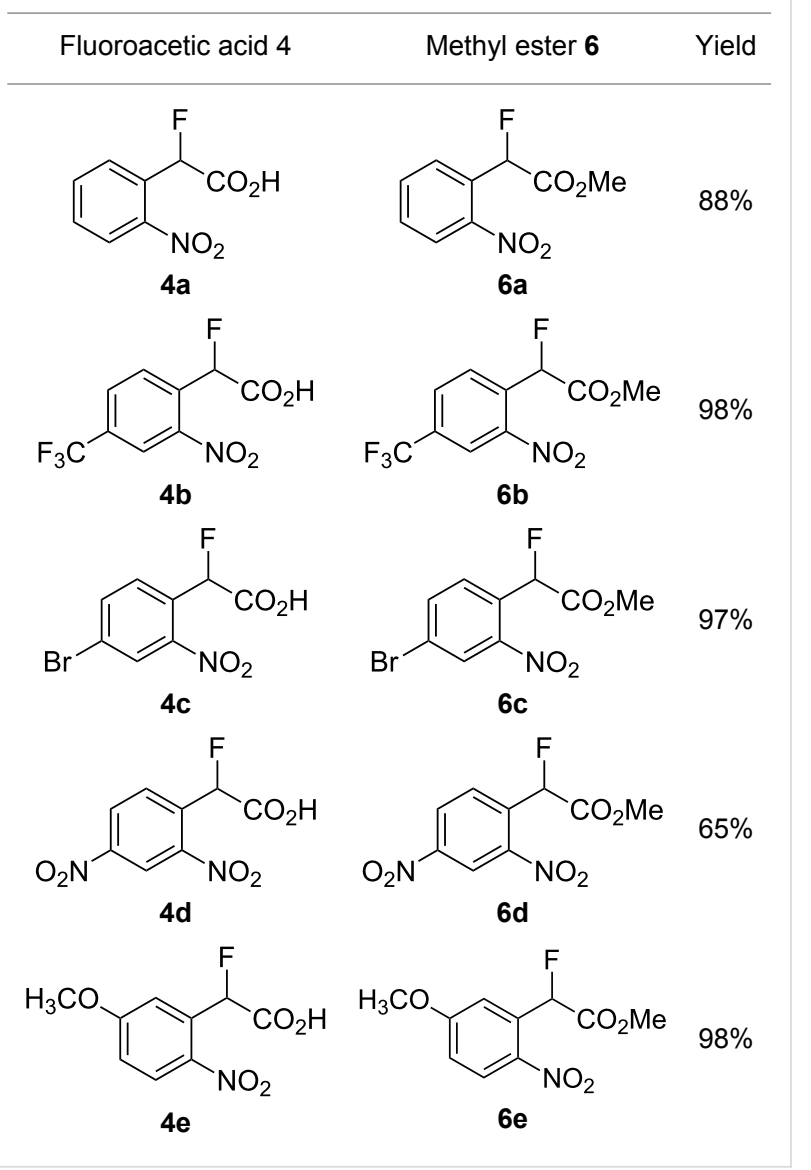

was confirmed unambiguously by X-ray crystallography (Figure 2) and all other methyl esters $\mathbf{6 b}-\mathbf{e}$ were characterised by comparison with appropriate NMR data obtained for $\mathbf{6 a}$.

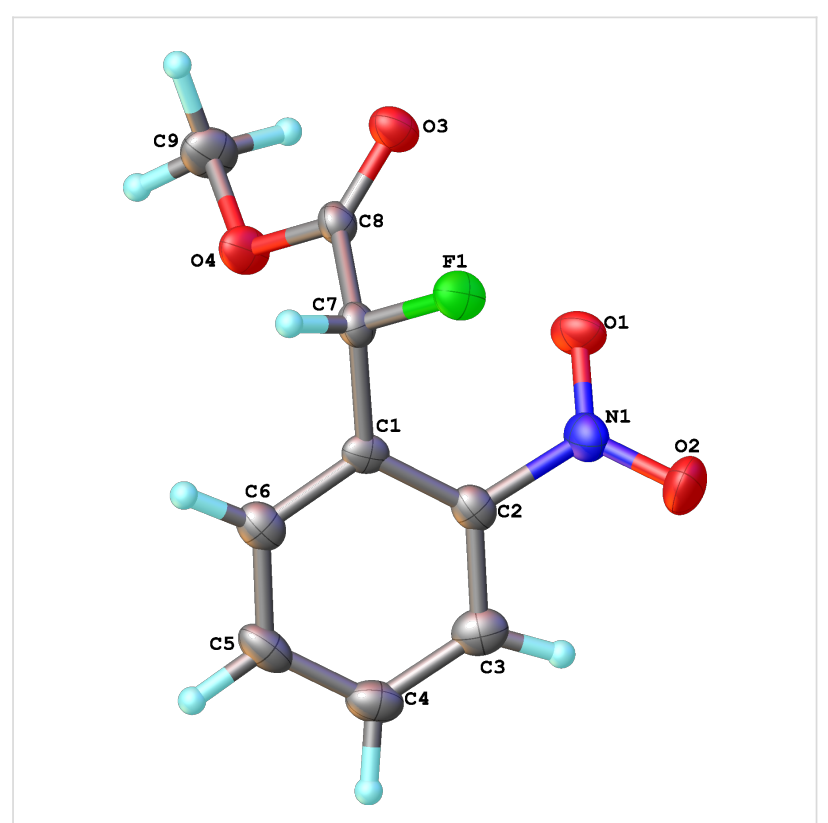

Figure 2: Molecular structure of methyl ester $\mathbf{6 a}$.

However, corresponding attempted esterification of the salt $\mathbf{4 f}$ with $\mathrm{HCl}$ in methanol gave 2-fluoromethyl-3-nitropyridine (7) in $68 \%$ yield (Scheme 3 ) after purification of the crude material by column chromatography and the structure was confirmed by X-ray analysis (Figure 3). In this case competing decarboxylation, rather than esterification, reflects the greater stabilisation of the carbanion system formed upon decarboxylation for this system.

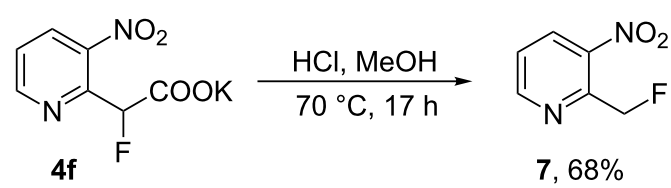

Scheme 3: Synthesis of pyridyl fluoride 7.

Reductive cyclization of methyl esters $\mathbf{6 a - e}$ using sodium dithionite provided fluorooxindoles $\mathbf{8 a}-\mathbf{e}$ in acceptable yield after isolation by column chromatography (Table 3 ). In the ${ }^{1} \mathrm{H}$ NMR spectrum, the characteristic CHF doublet located at $5.7 \mathrm{ppm}\left({ }^{2} J_{\mathrm{HF}}=51 \mathrm{~Hz}\right)$ for the fluorooxindole systems 8 are 0.9 ppm upfield from the corresponding CHF resonances of the arylfluoroacetic esters $\mathbf{6 a - e}$ and, additionally, a broad $\mathrm{NH}$ singlet was detected at $9.0 \mathrm{ppm}$. The chemical shift of the doublet $(-194.8 \mathrm{ppm})$ in the ${ }^{19} \mathrm{~F}$ NMR spectrum of fluorooxin- 


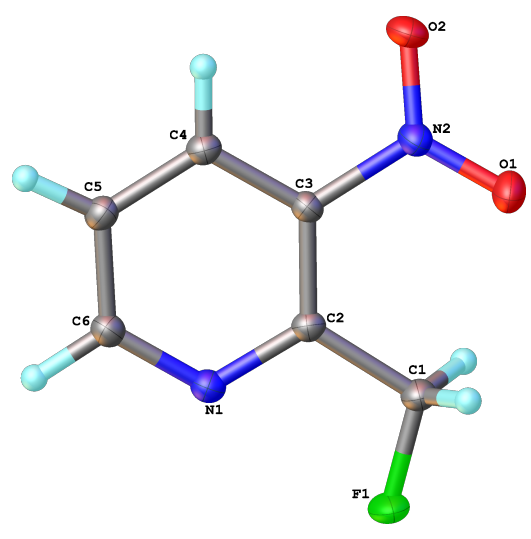

Figure 3: Molecular structure of 7.

Table 3: Synthesis of 3-fluorooxindoles.

$$
\begin{aligned}
& \overbrace{\mathrm{NO}_{2}}^{\mathrm{CO} M e} \\
& \text { 6a-e } \\
& \mathrm{Na}_{2} \mathrm{~S}_{2} \mathrm{O}_{4} \\
& \underset{\mathrm{THF}, \mathrm{H}_{2} \mathrm{O}}{\stackrel{\mathrm{NaHCO}_{3}}{\longrightarrow}}
\end{aligned}
$$

Methyl ester 6<smiles>CC(=O)C(F)c1ccccc1[N+](=O)[O-]</smiles>

$6 \mathbf{a}$<smiles>CC(=O)C(F)c1ccc(C(F)(F)F)cc1[N+](=O)[O-]</smiles>

$6 \mathrm{~b}$<smiles>CC(=O)C(F)c1ccc(Br)cc1[N+](=O)[O-]</smiles>

$6 c$<smiles>CC(=O)C(F)c1ccc([N+](=O)[O-])cc1[N+](=O)[O-]</smiles>

6d<smiles>COc1ccc([N+](=O)[O-])c(C(F)C(C)=O)c1</smiles>

$6 \mathrm{e}$<smiles>[X]c1ccc2c(c1)N([1H])C(=O)C2F</smiles>

8a-e

Fluorooxindole $8 \quad$ Yield

$\mathrm{F}_{3} \mathrm{C}$<smiles></smiles>

$8 \mathbf{a}$<smiles>O=C1Nc2cc(C(F)(F)F)ccc2C1F</smiles>

$8 b$<smiles>O=C1Nc2cc(Br)ccc2C1F</smiles>

8c<smiles>O=C1Nc2cc([N+](=O)[O-])ccc2C1F</smiles>

8d<smiles>COc1ccc2c(c1)C(F)C(=O)N2</smiles>

$8 e$
$32 \%$

$82 \%$

$57 \%$

$0 \%$

$30 \%$ doles 8a-e is also observed $10 \mathrm{ppm}$ upfield from the fluorine resonance of the starting esters $\mathbf{6 a}-\mathbf{e}$.

\section{Conclusion}

Diethyl 2-fluoromalonate ester can be used as a highly effective fluorinated building block for the synthesis of various polyfunctional 2-fluoroacetic acid and 3-fluorooxindole systems. Fluorooxindoles are relatively rare fluorinated heterocyclic systems, even though several derivatives have useful biological activity, and current literature syntheses only involve fluorination of appropriate hydroxy and oxindole substrates. The strategy described here provides complementary building block syntheses from readily available fluorinated starting materials, further demonstrating the viability of using fluorinated dicarbonyl systems for the synthesis of more structurally sophisticated fluorinated derivatives.

\section{Supporting Information}

\section{Supporting Information File 1}

Experimental procedures.

[http://www.beilstein-journals.org/bjoc/content/ supplementary/1860-5397-10-119-S1.pdf]

\section{Supporting Information File 2}

NMR spectra.

[http://www.beilstein-journals.org/bjoc/content/ supplementary/1860-5397-10-119-S2.pdf]

\section{Supporting Information File 3}

$\mathrm{X}$-ray crystallographic data.

[http://www.beilstein-journals.org/bjoc/content/ supplementary/1860-5397-10-119-S3.cif]

\section{References}

1. Fried, J.; Sabo, E. F. J. Am. Chem. Soc. 1954, 76, 1455-1456. doi:10.1021/ja01634a101

2. Müller, K.; Faeh, C.; Diedrich, F. Science 2007, 317, 1881-1886 doi:10.1126/science.1131943

3. Ojima, I., Ed. Fluorine in Medicinal Chemistry and Chemical Biology; Wiley-Blackwell: Oxford, 2009.

4. Purser, S.; Moore, P. R.; Swallow, S.; Gouverneur, V. Chem. Soc. Rev. 2008, 37, 320-330. doi:10.1039/b610213c

5. Isanbor, C.; O'Hagan, D. J. Fluorine Chem. 2006, 127, 303-319. doi:10.1016/j.jfluchem.2006.01.011

6. Kirk, K. L. J. Fluorine Chem. 2006, 127, 1013-1029. doi:10.1016/j.jfluchem.2006.06.007

7. Gribble, G. W. Chem. Soc. Rev. 1999, 28, 335-346. doi:10.1039/a900201d

8. Hall, R. J. New Phytol. 1972, 71, 855-871. doi:10.1111/j.1469-8137.1972.tb01965.x 
9. O'Hagan, D.; Schaffrath, C.; Cobb, S. L.; Hamilton, J. T. G.; Murphy, C. D. Nature 2002, 416, 279. doi:10.1038/416279a

10. Baasner, B.; Hagemann, H.; Tatlow, J. C. Houben-Weyl Organofluorine Compounds; Thieme: Stuttgart, 2000; Vol. E10a.

11. Chambers, R. D. Fluorine in Organic Chemistry; Blackwell: Oxford, 2004.

12. Uneyama, K. Organofluorine Chemistry; Blackwell: Oxford, 2006 doi:10.1002/9780470988589

13. Liang, T.; Neumann, C. N.; Ritter, T. Angew. Chem., Int. Ed. 2013, 52, 8214-8264. doi:10.1002/anie.201206566

14. Percy, J. M. Top. Curr. Chem. 1997, 199, 131-195. doi:10.1007/3-540-69197-9_4

15. Seebach, D. Angew. Chem., Int. Ed. Engl. 1990, 29, 1320-1367. doi:10.1002/anie.199013201

16. Warren, S. Designing Organic Syntheses: A Progrmmed Introduction to the Synthon Approach; John Wiley and Sons: New York, 1978.

17. Warren, S.; Wyatt, P. Organic Synthesis: The Disconnection Approach, 2nd ed.; John Wiley and Sons: Chichester, 2008.

18. Wyatt, P.; Warren, S. Organic Synthesis: Strategy and Control, 2nd ed.; John Wiley and Sons: Chichester, 2007.

19. Corey, E. J.; Cheng, X.-M. The logic of chemical synthesis; John Wiley and Sons: New York, 1995.

20. Chambers, R. D.; Hutchinson, J.; Thomson, J. J. Fluorine Chem. 1996, 78, 165-166. doi:10.1016/0022-1139(96)03422-7

21. Chambers, R. D.; Fox, M. A.; Holling, D.; Nakano, T.; Okazoe, T.; Sandford, G. Chem. Eng. Technol. 2005, 28, 344-352. doi:10.1002/ceat.200407123

22. Chambers, R. D.; Hutchinson, J. J. Fluorine Chem. 1998, 92, 45-52. doi:10.1016/S0022-1139(98)00254-1

23. Müh, T.; Fiedler, P.; Weintritt, H.; Westerkamp, W.; Reinecke, A. Method for producing alpha fluoromalonic acid dialkyl esters. W.O. Patent WO2002016304, Feb 28, 2002.

24. Günther, A.; Weintritt, H.; Böhm, S. Method for producing a-fluoromalonic acid dialkyl esters. W.O. Patent WO2005019154, March 3, 2005.

25. Braun, M.; Brosch, C. Production of fluorine compounds. W.O. Patent WO2002060838, Aug 8, 2002.

26. Ishikawa, N.; Takaoka, A. Chem. Lett. 1981, 10, 107-110. doi:10.1246/cl.1981.107

27. Ishikawa, N.; Takaoka, A.; Ibrahim, M. K. J. Fluorine Chem. 1984, 25, 203-212. doi:10.1016/S0022-1139(00)80949-5

28. Miller, T. A.; Sloman, D. L.; Stanton, M. G.; Wilson, K. J.; Witter, D. J. Fluorinated arylamide derivatives. W.O. Patent WO2007087129, Aug 2, 2007.

29. Close, J.; Heidebrecht, R. W.; Kattar, S.; Miller, T. A.; Sloman, D.; Stanton, M. G.; Tempest, P.; Witter, D. J. Histone deacetylase inhibitors with aryl-pyrazolyl motifs. W.O. Patent WO2007055941, May 18, 2007.

30. Hubbs, J. L.; Mampreian, D. M.; Methot, J. L.; Miller, T. A.; Otte, K. M.; Siliphaivanh, P.; Sloman, D. L.; Stanton, M. G.; Wilson, K. J.; Witter, D. J. Benzothiophene derivatives. W.O. Patent WO2006115845, Nov 2, 2006.

31. Bressi, J. C.; Chu, S.; Erickson, P.; Komandla, M.; Kwok, L.; Lawson, J. D.; Stafford, J. A.; Wallace, M. B.; Zhang, Z.; Das, J. cMET inhibitors. W.O. Patent WO2010019899, Feb 18, 2010.

32. Leonardi, A.; Riva, C.; Tavecchia, P.; Sironi, G. Aldosterone receptor antagonists. W.O. Patent WO2007025780, March 8, 2007.

33. Bergmann, E. D.; Cohen, S.; Shahak, I. J. Chem. Soc. 1959, 3286-3289. doi:10.1039/jr9590003286
34. Brickner, S. J.; Chen, J. M.; Li, Z. B.; Marfat, A.; Mitton-Fry, M. J.; Plotkin, M. A.; Reilly, U. D.; Subramanyam, C.; Zhang, Z.; Robinson, S. Substituted heterocyclic derivatives and their pharmaceutical use and compositions. U.S. Patent US20080280879, Nov 13, 2008.

35. Polla, M. O.; Tottie, L.; Nordén, C.; Linschoten, M.; Müsil, D.; Trumpp-Kallmeyer, S.; Aukrust, I. R.; Ringom, R.; Holm, K. H.; Neset, S. M.; Sandberg, M.; Thurmond, J.; Yu, P.; Hategan, G.; Anderson, H. Bioorg. Med. Chem. 2004, 12, 1151-1175. doi:10.1016/j.bmc.2003.12.039

36. Kim, D.-Y.; Kim, S.-M.; Koh, K.-O.; Mang, J.-Y.; Lee, K.-S. Bull. Korean Chem. Soc. 2003, 24, 1425-1426. doi:10.5012/bkcs.2003.24.10.1425

37. Cho, M.-J.; Cho, M.-G.; Huh, S.-C.; Kim, S.-M.; Lee, K.-s.; Koh, K.-O.; Mang, J.-Y.; Kim, D.-Y. Bull. Korean Chem. Soc. 2006, 27, 857-862. doi:10.5012/bkcs.2006.27.6.857

38. Kang, S.-H.; Kim, D.-Y. Bull. Korean Chem. Soc. 2009, 30, 1439-1440. doi:10.5012/bkcs.2009.30.7.1439

39. Kwon, B. K.; Kim, S. M.; Kim, D. Y. J. Fluorine Chem. 2009, 130, 759-761. doi:10.1016/j.jfluchem.2009.06.002

40. Li, H.; Zu, L.; Xie, H.; Wang, W. Synthesis 2009, 1525-1530. doi:10.1055/s-0028-1088124

41. Sandford, G. J. Fluorine Chem. 2007, 128, 90-104. doi:10.1016/j.jfluchem.2006.10.019

42. Chambers, R. D.; Nakano, T.; Parsons, M.; Sandford, G.; Batsanov, A. S.; Howard, J. A. K. J. Fluorine Chem. 2008, 129 , 811-816. doi:10.1016/j.jfluchem.2008.04.010

43. Chambers, R. D.; Sandford, G.; Trmcic, J.; Okazoe, T. Org. Process Res. Dev. 2008, 12, 339-344. doi:10.1021/op700194r

44. Chambers, R. D.; Nakano, T.; Okazoe, T.; Sandford, G. J. Fluorine Chem. 2009, 130, 792-798. doi:10.1016/j.jfluchem.2009.06.003

45. Chambers, R. D.; Fox, M. A.; Holling, D.; Nakano, T.; Okazoe, T.; Sandford, G. Lab Chip 2005, 5, 191-198. doi:10.1039/b416400h

46. Chambers, R. D.; Fox, M. A.; Holling, D.; Nakano, T.; Okazoe, T.; Sandford, G. Chem. Eng. Technol. 2005, 28, 344-352. doi:10.1002/ceat.200407123

47. Chambers, R. D.; Fox, M. A.; Sandford, G. Lab Chip 2005, 5, 1132-1139. doi:10.1039/b504675k

48. Breen, J. R.; Sandford, G.; Yufit, D. S.; Howard, J. A. K.; Fray, J.; Patel, B. Beilstein J. Org. Chem. 2011, 7, 1048-1054. doi:10.3762/bjoc.7.120

49. McPake, C. B.; Sandford, G. Org. Process Res. Dev. 2012, 16, 844-851. doi:10.1021/op200331s

50. Hutchinson, J.; Sandford, G.; Vaughan, J. F. S. Tetrahedron 1998, 54, 2867-2876. doi:10.1016/S0040-4020(98)83023-8

51. Beare, N. A.; Hartwig, J. F. J. Org. Chem. 2002, 67, 541-555. doi:10.1021/j0016226h

52. Harsanyi, A. M.Sc. Thesis, Synthesis of 3-fluoro-oxindoles and phenyl fluoroacetic acid derivatives; Durham University, 2013, available from http://etheses.dur.ac.uk/6357/.

53. Shibata, N.; Suzuki, E.; Asahi, T.; Shiro, M. J. Am. Chem. Soc. 2001, 123, 7001-7009. doi:10.1021/ja010789t

54. Shibata, N.; Ishimaru, T.; Suzuki, E.; Kirk, K. L. J. Org. Chem. 2003, 68, 2494-2497. doi:10.1021/jo026792s

55. Zoute, L.; Audouard, C.; Plaquevent, J.-C.; Cahard, D. Org. Biomol. Chem. 2003, 1, 1833-1834. doi:10.1039/b303113f

56. Shibata, N.; Kohno, J.; Takai, K.; Ishimaru, T.; Nakamura, S.; Toru, T.; Kanemasa, S. Angew. Chem., Int. Ed. 2005, 44, 4204-4207. doi:10.1002/anie.200501041 
57. Hamashima, Y.; Suzuki, N.; Takano, H.; Shimura, Y.; Sodeoka, M. J. Am. Chem. Soc. 2005, 127, 10164-10165. doi:10.1021/ja0513077

58. Deng, Q.-H.; Wadepohl, H.; Gade, L. H. Chem.-Eur. J. 2011, 17, 14922-14928. doi:10.1002/chem.201102375

59. Li, J.; Cai, Y.; Chen, W.; Liu, X.; Lin, L.; Feng, X. J. Org. Chem. 2012, 77, 9148-9155. doi:10.1021/jo301705t

60. Wu, L.; Falivene, L.; Drinkel, E.; Grant, S.; Linden, A.; Cavallo, L.; Dorta, R. Angew. Chem., Int. Ed. 2012, 51, 2870-2873. doi:10.1002/anie.201200206

61. Ishimaru, T.; Shibata, N.; Horikawa, T.; Yasuda, N.; Nakamura, S.; Toru, T.; Shiro, M. Angew. Chem., Int. Ed. 2008, 47, 4157-4161. doi:10.1002/anie.200800717

62. Dou, X.; Lu, Y. Org. Biomol. Chem. 2013, 11, 5217-5221. doi:10.1039/c3ob41267a

63. Hewawasam, P.; Gribkoff, V. K.; Pendri, Y.; Dworetzky, S. I.; Meanwell, N. A.; Martinez, E.; Boissard, C. G.; Post-Munson, D. J.; Trojnacki, J. T.; Yeleswaram, K.; Pajor, L. M.; Knipe, J.; Gao, Q.; Perrone, R.; Starret, J. E. Bioorg. Med. Chem. Lett. 2002, 12, 1023-1026. doi:10.1016/S0960-894X(02)00101-4

64. Schiefer, I. T.; Abdul-Hay, S.; Wang, H.; Vanni, M.; Qin, Z.; Thatcher, G. R. J. J. Med. Chem. 2011, 54, 2293-2306. doi:10.1021/jm101450p

65. Zhang, F.; Song, J. Z. Tetrahedron Lett. 2006, 47, 7641-7644. doi:10.1016/j.tetlet.2006.08.057

66. Rozen, S.; Hagooly, A.; Harduf, R. J. Org. Chem. 2001, 66, 7464-7468. doi:10.1021/jo010677k

67. Davis, F. A.; Han, W.; Murphy, C. K. J. Org. Chem. 1996, 60, 4730-4737. doi:10.1021/jo00120a014

68. Cantrell, G. L.; Filler, R. J. Fluorine Chem. 1985, 27, 35-45. doi:10.1016/S0022-1139(00)80895-7

69. Bresciani, S.; O'Hagan, D. Tetrahedron Lett. 2010, 51, 5795-5797. doi:10.1016/j.tetlet.2010.08.104

70. Watanabe, S.; Fujita, T.; Sakamoto, M.; Endo, H.; Kitazume, T. J. Fluorine Chem. 1990, 47, 187-192. doi:10.1016/S0022-1139(00)82371-4

71. Kim, K.-Y.; Kim, B. C.; Lee, H. B.; Shin, H. J. Org. Chem. 2008, 73, 8106-8108. doi:10.1021/jo8015659

72. Yin, J.; Zarkowsky, D. S.; Thomas, D. W.; Zhao, M. M.; Huffman, M. A. Org. Lett. 2004, 6, 1465-1468. doi:10.1021/ol049672a

\section{License and Terms}

This is an Open Access article under the terms of the Creative Commons Attribution License (http://creativecommons.org/licenses/by/2.0), which permits unrestricted use, distribution, and reproduction in any medium, provided the original work is properly cited.

The license is subject to the Beilstein Journal of Organic Chemistry terms and conditions: (http://www.beilstein-journals.org/bjoc)

The definitive version of this article is the electronic one which can be found at: doi:10.3762/bjoc. 10.119 